\title{
Scattered-wave-packet formalism with applications to barrier scattering and quantum transistors
}

\author{
Chia-Chun Chou* and Robert E. Wyatt ${ }^{\dagger}$ \\ Institute for Theoretical Chemistry and Department of Chemistry and Biochemistry, The University of Texas at Austin, \\ Austin, Texas 78712, USA
}

(Received 9 June 2011; published 23 November 2011)

\begin{abstract}
The scattered wave formalism developed for a quantum subsystem interacting with reservoirs through open boundaries is applied to one- or two-dimensional barrier scattering and quantum transistors. The total wave function is divided into incident and scattered components. Markovian outgoing wave boundary conditions are imposed on the scattered or total wave function by either the ratio or polynomial methods. For barrier scattering problems, accurate time-dependent transmission probabilities are obtained through the integration of the modified time-dependent Schrödinger equations for the scattered wave function. For quantum transistors, the time-dependent transport is studied for a quantum wave packet propagating through the conduction channel of a field effect transistor. This study shows that the scattered wave formalism significantly reduces computational effort relative to other open boundary methods and demonstrates wide applications to quantum dynamical processes.
\end{abstract}

DOI: 10.1103/PhysRevE.84.056707

PACS number(s): 02.70.-c, 03.65.Nk, 73.63.-b, 85.30.Tv

\section{INTRODUCTION}

When solving the time-dependent Schrödinger equation (TDSE) in a finite computational region, great care must be exercised to avoid spurious reflections of the reflected and transmitted waves at the boundaries. Various approaches have been developed to mimic a spatial domain of infinite extent using boundary conditions at the edges of a finite computational grid. For example, absorbing potentials at the edges of grids can be used but they may perturb the dynamics of the solution near the boundary [1-3]. Exact non-Markovian open-system boundary conditions, exact boundary conditions at finite distance, and discrete transparent boundary conditions have been formulated for outgoing solutions of the TDSE [4-6]. However, these methods are either too complicated to be implemented or their evaluation is too time consuming for complex multidimensional quantum systems.

In our previous study [7], we developed the scattered wave formalism with Markovian outgoing wave boundary conditions for open quantum systems. In this formalism, the total wave function is split into the incident and scattered parts, and outgoing wave boundary conditions are applied to the scattered wave function. The scattered wave function describes the outgoing waves generated by the interaction between the incident wave and the potential. Assuming the scattered wave function has the form of an outgoing wave near open boundaries, we derived a simple equation relating the scattered wave function at the boundary to the scattered wave function at interior points. Thus, the scattered wave function at a few interior grid points can be used to determine the scattered wave function at the boundary. Here, these boundary conditions are not treated using negative imaginary absorbing potentials, Green functions, or memory kernels, and they are Markovian boundary conditions which require only the form of the scattered wave at the current time in the internal region near the open boundaries. Therefore, the scattered wave function

\footnotetext{
*chiachun@mail.utexas.edu

†wyattre@mail.utexas.edu
}

significantly reduces computational effort relative to other open boundary methods.

The scattered wave formalism has been applied to the scattering of a Gaussian wave packet from a symmetric Eckart barrier and to the three-dimensional field effect transistor [7]. In this study, we first propose another method, called the polynomial method, for imposing open boundary conditions at the edges of the system. This method employs the least squares method to fit the scattered or total wave functions at the boundary by extrapolation. In addition, we extend our previous analysis to barrier scattering, including a general onedimensional potential barrier with different asymptotic energies and a two-dimensional system involving an Eckart barrier along the reaction coordinate coupled to a harmonic oscillator.

Moreover, the scattered wave formalism will be applied to the nanoscale field effect transistor (FET). In this device, the electron current flowing from the source to the drain is modulated by passage through a gate region where the electric potential can be altered. Due to the very small length of the conduction channel, quantum effects become significant and the TDSE must be solved in the conduction channel with open boundary conditions applied at interfaces between the device and the external contacts. The quantum dynamics will be studied for wave packets propagating through the channel for specified gate and drain voltages (the source lead is grounded). A computational grid will be introduced in the channel and open boundary conditions will be applied at the source-channel and drain-channel contacts. Any form of incident wave packet can be considered, but in this study the incident current pulse is a Gaussian wave packet. Accurate computational results for these examples illustrate the stability, efficiency, and accuracy of the open boundary conditions used in the scattered wave formalism. Therefore, the current study demonstrates that the scattered wave formalism is suitable for studying various time-dependent quantum dynamical processes.

The organization of the remainder of this study is as follows. In Sec. II, we briefly introduce the scattered wave formalism and the ratio and polynomial methods for imposing open boundary conditions. Then, the scattered wave formalism 
is applied to barrier scattering in Sec. III and to quantum transistors in Sec. IV. In Sec. V, we summarize our results, conclude with some comments, and suggest directions for further research in this field.

\section{SCATTERED WAVE FORMALISM}

\section{A. Modified time-dependent Schrödinger equation for the scattered wave function}

The TDSE governing the time evolution of a quantum system is given by

$$
i \hbar \frac{\partial \Psi(\mathbf{r}, t)}{\partial t}=-\frac{\hbar^{2}}{2 m} \nabla^{2} \Psi(\mathbf{r}, t)+V(\mathbf{r}) \Psi(\mathbf{r}, t) .
$$

In the scattered wave formalism, we separate the total wave function into incident and scattered parts,

$$
\Psi(\mathbf{r}, t)=\Phi(\mathbf{r}, t)+\Upsilon(\mathbf{r}, t),
$$

and the initial condition for the wave function is given by $\Psi(\mathbf{r}, 0)=\Phi(\mathbf{r}, 0)$. We assume that the incident wave function satisfies the free-space TDSE

$$
i \hbar \frac{\partial \Phi(\mathbf{r}, t)}{\partial t}=-\frac{\hbar^{2}}{2 m} \nabla^{2} \Phi(\mathbf{r}, t) .
$$

The incident wave function describes a wave propagating in free space with the same initial condition as that for the total wave function. Substituting the decomposition of the total wave function in Eq. (2) and the free-space TDSE in Eq. (3) into the TDSE for the total wave function in Eq. (1), we obtain the modified TDSE for the scattered wave function

$$
i \hbar \frac{\partial \Upsilon(\mathbf{r}, t)}{\partial t}=-\frac{\hbar^{2}}{2 m} \nabla^{2} \Upsilon(\mathbf{r}, t)+V(\mathbf{r}) \Upsilon(\mathbf{r}, t)+V(\mathbf{r}) \Phi(\mathbf{r}, t),
$$

where the initial condition is given by $\Upsilon(\mathbf{r}, 0)=0$. As indicated in this equation, the scattered wave function is initially equal to zero, and then the scattered wave is generated by the interaction between the incident wave and the potential. Thus, the last term in the modified TDSE serves as an inhomogeneous source term or a driving term for the scattered wave function. The scattered wave function describes the scattered outgoing waves created in the interaction region of the potential. In general, the incident wave function can be easily obtained by solving the free-space TDSE in Eq. (3). Then, substituting the incident wave function into the source term in Eq. (4), we can solve the modified TDSE for the scattered wave function. The total wave function is given by the sum of the incident and scattered wave functions.

\section{B. Boundary conditions for the scattered wave function}

\section{Ratio method for imposing boundary conditions}

It seems that there is no advantage to solving two equations (the free-space TDSE and the modified TDSE) instead of directly solving the TDSE. However, open boundary conditions can be applied to the scattered wave function to significantly reduce the size of the computational domain. Without loss of generality, we consider the one-dimensional case. First, the computational domain $[\alpha, \beta]$ for the scattered wave function is discretized by $N+1$ grid points $x_{n}=n \Delta x$ with $x_{0}=\alpha$ and $x_{N}=\beta$. Outgoing wave boundary conditions are applied to the scattered wave function at the left and right boundaries. At the left boundary, the scattered wave function is assumed to have the form of an outgoing wave, $\Upsilon(x)=\Upsilon_{0} \exp (-i k x)$, provided that we have a constant potential near the boundaries of the computational domain. Here, the coefficient $\Upsilon_{0}$ and the wave vector $k$ may be complex valued. Thus, the scattered wave function at the left three grid points are given by $\Upsilon\left(x_{0}\right)=\Upsilon_{0} \exp \left(-i k x_{0}\right)$, $\Upsilon\left(x_{1}\right)=\Upsilon_{0} \exp \left(-i k x_{1}\right)$, and $\Upsilon\left(x_{2}\right)=\Upsilon_{0} \exp \left(-i k x_{2}\right)$, where $x_{1}=x_{0}+\Delta x$ and $x_{2}=x_{0}+2 \Delta x$. From these equations, we can derive an equation associated with the scattered wave at the left three grid points,

$$
\Upsilon\left(x_{0}\right)=\frac{\Upsilon\left(x_{1}\right)^{2}}{\Upsilon\left(x_{2}\right)} .
$$

Thus, the scattered wave function at the interior grid points $x_{1}$ and $x_{2}$ can be used to determine the scattered wave function at the left boundary. Analogously, the scattered wave function is assumed to have the form of an outgoing wave at the right boundary, $\Upsilon(x)=\Upsilon_{0} \exp (i k x)$. We can derive a similar condition for the scattered wave function

$$
\Upsilon\left(x_{N}\right)=\frac{\Upsilon\left(x_{N-1}\right)^{2}}{\Upsilon\left(x_{N-2}\right)} \text {. }
$$

The scattered wave function at the interior grid points $x_{N-2}$ and $x_{N-1}$ can be used to determine the scattered wave function at the right boundary. In addition, the ratio method for the outgoing wave boundary conditions has previously been imposed on the numerical solution of the TDSE for the total wave function to study the decay dynamics of low-lying unbound proton states and deep tunneling during proton emission from nuclei [8-10].

\section{Polynomial method for imposing boundary conditions}

The second method for imposing open boundary conditions at the edges of the system also requires only information on the internal wave function close to the boundaries. In order to simplify the notation, the polynomial method will be introduced for a one-dimensional example. Assume again that the system grid points are labeled $x_{0}$ to $x_{N}$ and that the wave function has been advanced one time step at the internal grid points $x_{1}$ to $x_{N-1}$. The problem again is how to update values of the wave function at the two boundary points. The method is introduced by invoking the polar decomposition of the wave function, $\psi(x, t)=R(x, t) \exp [i \phi(x, t)]$, where $R$ and $\phi$ are the amplitude and phase, respectively. The amplitude satisfies the condition $R \geqslant 0$ and the phase is continuous and single valued. At the internal grid points, the amplitude is obtained from the equation $R=\left(\psi^{*} \psi\right)^{1 / 2}$ and the phase is given by $\phi=-i \ln (\psi / R)$. However, the latter relation usually returns a jagged saw-toothed function with values in the range $-\pi$ to $\pi$, but these segments may be linked together to produce a continuous function. (In MATLAB, these two operations may be performed with the functions angle and unwrap.) At this stage, we have the continuous functions $R(x, t)$ and $\phi(x, t)$ at the internal grid points. The basis for the polynomial method described below is that even when $\psi(x, t)$ is rapidly oscillating, $R$ and $\phi$ are frequently slowly varying with respect to the $x$ coordinate. 
Near the system boundaries, $R$ and $\phi$ can be least squares fit to a polynomial with a low degree (denoted by $n$ ),

$$
R(x)=\sum_{j=0}^{n} a_{j}\left(x-x_{0}\right)^{j} \quad \text { and } \quad \phi(x)=\sum_{j=0}^{n} b_{j}\left(x-x_{0}\right)^{j} .
$$

These fits utilize the known values of $R$ and $\phi$ at $n_{p}$ internal grid points located near the left boundary. For least squares fitting, it is required that $n_{p}>n+1$. Then, using these fits, function values at the boundary are obtained by extrapolation, $R\left(x_{0}\right)=a_{0}$ and $\phi\left(x_{0}\right)=b_{0}$. (In MATLAB, least squares fitting and extrapolation may be performed with the functions polyfit and polyval.) A similar procedure yields the amplitude and phase at the right boundary. For the computations reported in Sec. IV C, linear fits with input data at four points were used: $n=1$ and $n_{p}=4$. If we use these values for the fitting parameters, the wave function near the left boundary has the form

$$
\psi(x, t)=\left\{\left[a_{0}+a_{1}\left(x-x_{0}\right)\right] e^{i\left[b_{0}+b_{1}\left(x-x_{0}\right)\right]}\right\} .
$$

Of course, higher-order fits may be used in Eq. (7), but the computational effort increases correspondingly.

The relationship between the polynomial and ratio methods is readily obtained. Using Eq. (8), at the first two internal points $x_{0}+\delta$ and $x_{0}+2 \delta$ we obtain the ratio

$$
\frac{\psi\left(x_{1}\right)^{2}}{\psi\left(x_{2}\right)}=\frac{\left(a_{0}+\delta a_{1}\right)^{2}}{\left(a_{0}+2 \delta a_{1}\right)} e^{i b_{0}} .
$$

In addition, since Eq. (8) also gives $\psi\left(x_{0}\right)=a_{0} \exp \left(i b_{0}\right)$, we observe that the right side of Eq. (9) is equal to $\psi\left(x_{0}\right)$ only when $a_{1}=0$. Thus, we conclude that the ratio $\psi\left(x_{1}\right)^{2} / \psi\left(x_{2}\right)$ yields $\psi\left(x_{0}\right)$ when the amplitude $R(x)$ is constant (or slowly varying) near the boundary. In this sense, the polynomial method is more general than the ratio method for imposing boundary conditions on the wave function.

\section{Scattered wave propagation}

We summarize the procedure for the scattered wave formalism. First, the total wave function is separated into the incident and scattered parts. The incident wave function is determined either by analytically solving the free-space TDSE in Eq. (3) or by numerically solving this equation on a computational grid with the initial condition $\Phi(\mathbf{r}, 0)=\Psi(\mathbf{r}, 0)$. The incident wave function serves as a source term in the modified TDSE in Eq. (4) for the scattered wave function. Then, the scattered wave function is obtained on a reduced computational grid involving only the interaction region of the potential with open boundary conditions through the ratio or polynomial methods. At each time step, the scattered wave function at the interior grid points is employed to determine the scattered wave function at the boundaries. Finally, the sum of the incident and scattered wave functions gives the time evolution of the total wave function on the reduced computational domain.

\section{COMPUTATIONAL RESULTS FOR BARRIER SCATTERING}

In our previous study [7], the scattered wave formalism was illustrated for Gaussian wave-packet scattering from a symmetric Eckart barrier where the potential barrier approaches zero when $x$ tends to $\pm \infty$. In this section, the scattered wave formalism will be applied to Gaussian wave-packet scattering from a general asymmetric one-dimensional potential barrier with different asymptotic energies. Moreover, this formalism will be extended to a two-dimensional system involving an Eckart barrier along the reaction coordinate coupled to a harmonic oscillator.

\section{A. Asymmetric one-dimensional potential barrier}

We consider Gaussian wave-packet scattering from an asymmetric potential barrier given by

$$
V(x)=V_{0}\left[1-\left(\frac{1-e^{a x}}{1+c e^{a x}}\right)^{2}\right],
$$

where $V_{0}=1.0, a=2.5$, and $c=0.9$ (see Fig. 1). All quantities are given in atomic units $(m=1$ and $\hbar=1)$. The initial Gaussian wave packet is given by

$$
\Phi(x, 0)=\left(\frac{2 \beta}{\pi}\right)^{1 / 4} \exp \left[-\beta\left(x-x_{0}\right)^{2}+i \frac{p_{0}}{\hbar}\left(x-x_{0}\right)\right],
$$

where $\beta=0.6$ and $x_{0}=-7.0$. The incident wave function satisfying the initial condition and the free-space TDSE is
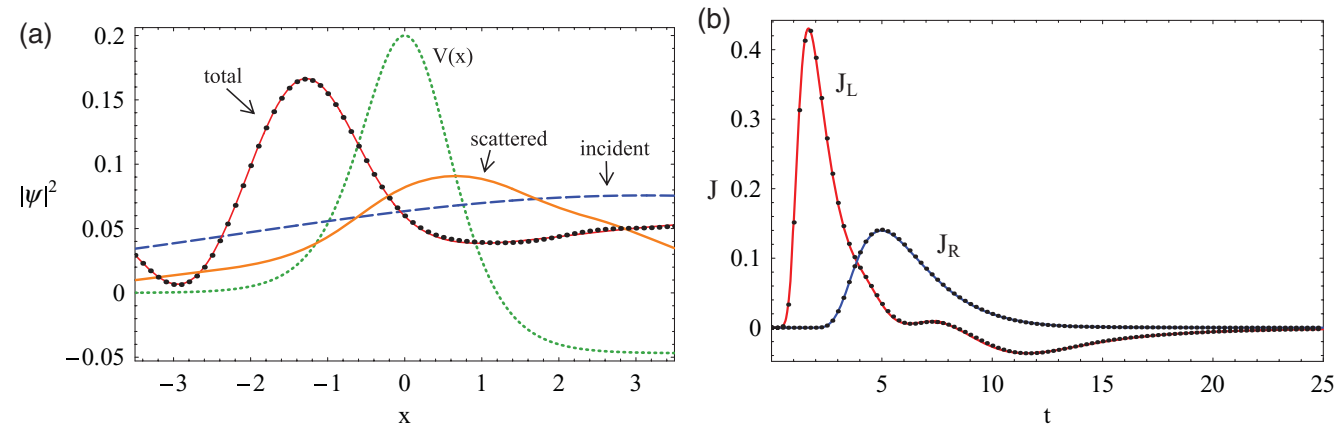

FIG. 1. (Color online) (a) The incident wave function (dashed line), the scattered wave function (solid line), the total wave function obtained from the scattered wave formalism (dots), and the total wave function from the integration of the TDSE using a large spatial grid (curves) with the one-dimensional potential barrier in arbitrary units (dotted line) at $t=6.75$. (b) The probability flux at the left and right boundaries obtained from the scattered wave formalism (dots) and from the integration of the TDSE using a large spatial grid (curves). 
known analytically [11],

$$
\begin{aligned}
\Phi(x, t)= & \left(\frac{2 \beta}{\pi}\right)^{1 / 4} \frac{1}{(1+2 i \beta \hbar t / m)^{1 / 2}} \\
& \times \exp \left[i \frac{p_{0}}{\hbar}\left(x-x_{0}-\frac{p_{0} t}{2 m}\right)\right. \\
& \left.-\frac{\beta\left(x-x_{0}-p_{0} t / m\right)^{2}}{(1+2 i \beta \hbar t / m)}\right] .
\end{aligned}
$$

This analytical expression was used in the source term for the integration of the modified TDSE in Eq. (4).

We solved the modified TDSE in Eq. (4) for the scattered wave function from $t=0$ to $t=25$ using the fourth-order Runge-Kutta method [12] with $\Delta t=0.005$ by applying the ratio method in Eqs. (5) and (6) to the boundaries. The computational domain was chosen so that $\left(x_{0}, x_{N}\right)=$ $(-3.5,3.5)$ with the spacing $\Delta x=0.1$. Figure 1(a) displays the probability densities at $t=6.75$ with $p_{0}=1.5$ for the incident, scattered, and total wave functions. As shown in this figure, the wave packet has split into reflected and transmitted parts, and the scattered wave function propagates through the boundaries without any reflection. Also, the total wave function $(\Psi=\Phi+\Upsilon)$ resulting from the sum of the incident and scattered wave functions is in excellent agreement with the exact result obtained by solving the TDSE using a large spatial grid without employing open boundary conditions.

Figure 1(b) shows the time-dependent probability flux at the left and right boundaries obtained from the scattered wave formalism. The time-dependent probability flux is defined by

$$
J(x, t)=\frac{\hbar}{m} \operatorname{Im}\left[\Psi^{*}(x, t) \frac{\partial \Psi(x, t)}{\partial x}\right] .
$$

Here, the probability flux at the left and right boundaries were calculated using the fourth-order forward and backward finite difference formulas to approximate the spatial derivative in Eq. (13) [12]. Initially, the Gaussian wave packet starts outside the computational domain. The wave packet enters the computational domain from the left boundary, and this leads to an increase in the probability flux $\left(J_{L}\right)$. When the incident wave packet begins to interact with the potential barrier, the reflected and transmitted wave packets start to form. As time progresses, the transmitted wave packets pass through the right boundary, and this leads to the positive flux $\left(J_{R}\right)$ from about $t=3$ to $t=12$. Analogously, the reflected wave packet travels through the left boundary, and this results in the negative flux from about $t=7$ to $t=20$. Finally, the probability flux at the two boundaries gradually decays to zero. This means that these two wave packets completely leave the computational grid without reflecting from the boundaries. As shown in this figure, the probability flux obtained from the scattered wave formalism is in excellent agreement with the exact result obtained from the solution of the TDSE using a large spatial grid without employing open boundary conditions.

The time-dependent transmission probability of a Gaussian wave-packet scattering from the potential barrier is defined by

$$
P(t)=\int_{0}^{\infty} \rho(x, t) d x
$$

where the probability density is given by $\rho(x, t)=|\psi(x, t)|^{2}$. The integration begins at $x=0$ because this is the position of the maximum of the barrier. Since we calculated the total wave function on a reduced computational domain with open boundary conditions, we cannot use Eq. (14) to evaluate the time-dependent transmission probability. However, the time-dependent transmission probability can be expressed by a time integration of the probability flux at $x=0$. Using the continuity equation for the probability density, we can write

$$
\begin{aligned}
P(t) & =\int_{0}^{\infty} \rho\left(x^{\prime}, t\right) d x^{\prime}=\int_{0}^{\infty} \int_{0}^{t} \frac{\partial \rho\left(x^{\prime}, t^{\prime}\right)}{\partial t^{\prime}} d t^{\prime} d x^{\prime} \\
& =-\int_{0}^{t} \int_{0}^{\infty} \frac{\partial J\left(x^{\prime}, t^{\prime}\right)}{\partial x^{\prime}} d x^{\prime} d t^{\prime}=\int_{0}^{t} J\left(x^{\prime}=0, t^{\prime}\right) d t^{\prime},
\end{aligned}
$$

where we changed the order of the integration and performed the spatial integration. Therefore, the time integration of the probability flux at $x=0$ gives the time-dependent transmission probability.

Figure 2 presents the time-dependent transmission probabilities of the initial Gaussian wave packet with three different values of $p_{0}$. Here, the probability flux at $x=0$ was calculated using the fourth-order central finite difference formulas to approximate the spatial derivative in Eq. (13) [12]. This figure indicates that the scattered wave formalism yields transmission probabilities evaluated by Eq. (15) in excellent agreement with the exact results obtained by solving the TDSE on a large grid $(x=[-50,100])$ without employing open boundary conditions. Therefore, the scattered wave formalism gives the excellent results by solving the modified TDSE on a very small computational grid.

\section{B. Two-dimensional barrier scattering}

We now consider a two-dimensional system with the potential energy given by

$$
V(x, y)=V_{0} \operatorname{sech}^{2}(2 x)+\frac{1}{2} k(x) y^{2},
$$

where $x$ and $y$ denote the translational and vibrational coordinates, respectively. The potential energy is an Eckart barrier of height $V_{0}$ centered at $x=0$, which is coupled to a

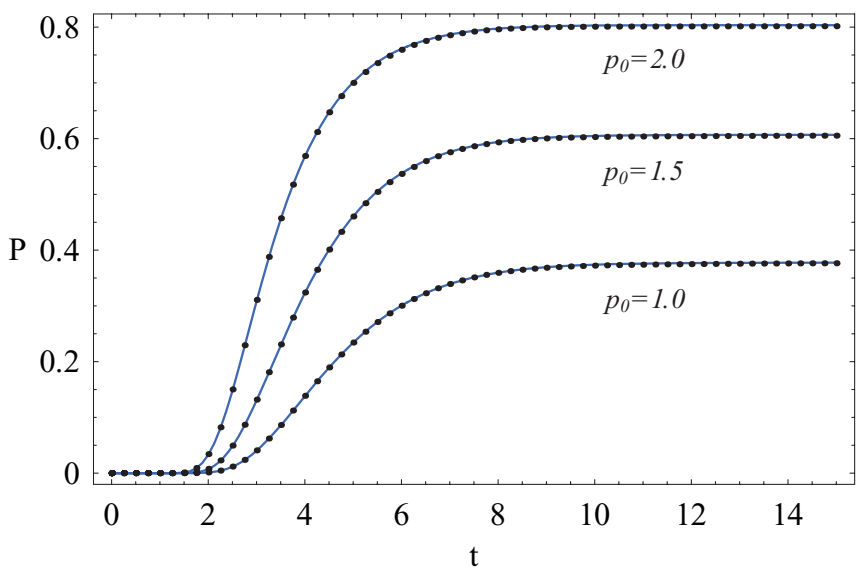

FIG. 2. (Color online) Time-dependent transmission probabilities obtained by the scattered wave formalism (dots) compared with the exact results (curves). 

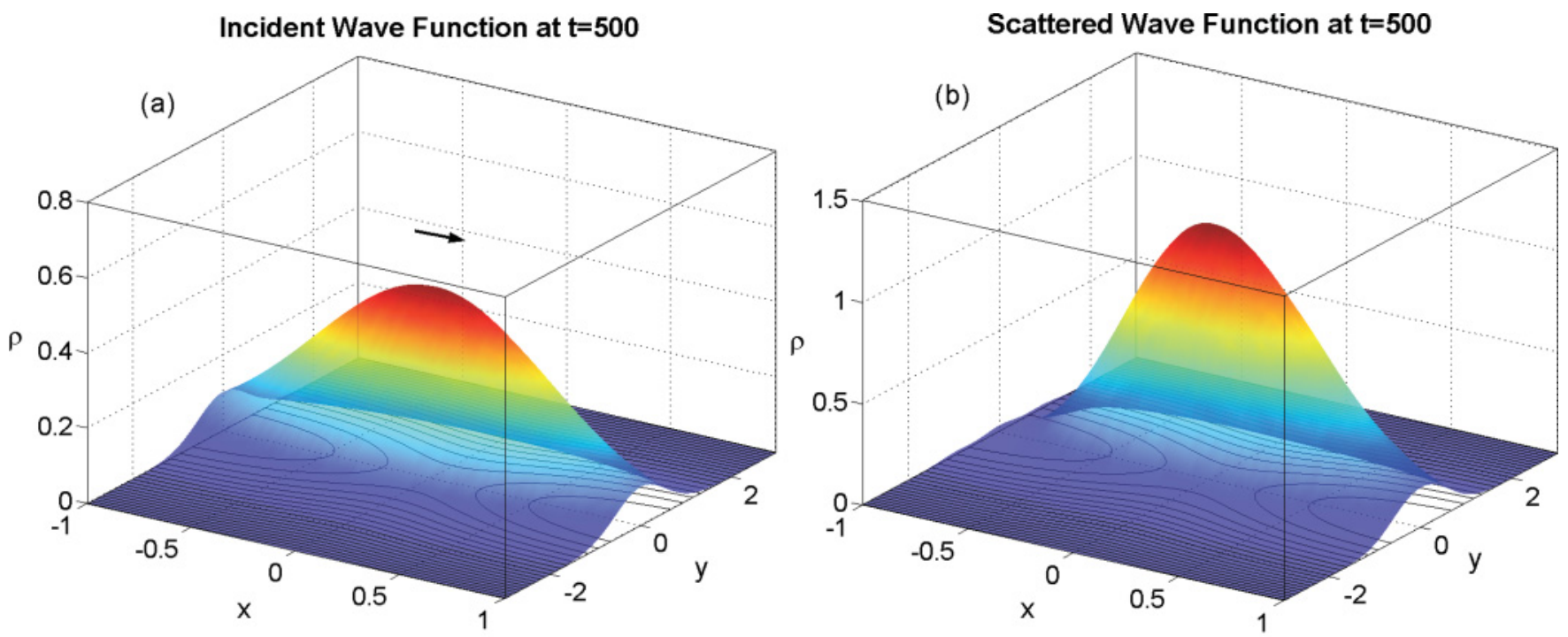

Total Wave Function at $\mathrm{t}=\mathbf{5 0 0}$

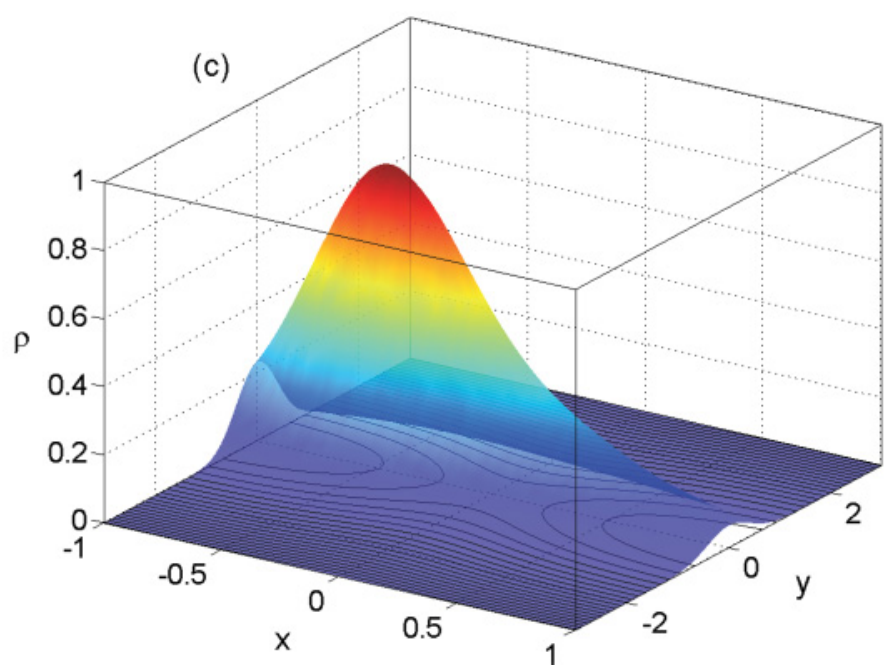

FIG. 3. (Color online) The incident, scattered, and total wave functions at $t=500$ for $k_{x}=4$ with contours of the potential barrier. The arrow indicates the propagation direction of the incident wave function.

harmonic oscillator with a variable force constant along $x$. The variable force constant $k(x)=k_{0}\left[1-\sigma \exp \left(-2 x^{2}\right)\right]$ reaches its minimum value, $k_{0}(1-\sigma)$, at the barrier maximum $(x=0)$. The values of the parameters are given as $V_{0}=0.00625$, $k_{0}=0.0106$, and $\sigma=0.1$. All quantities are given in atomic units $(\hbar=1)$. The initial Gaussian wave packet is given by

$$
\Phi(x, y, 0)=\left(\frac{4 \beta_{x} \beta_{y}}{\pi^{2}}\right)^{1 / 4} e^{\left[-\beta_{x}\left(x-x_{c}\right)^{2}-\beta_{y} y^{2}+i k_{x}\left(x-x_{c}\right)\right]},
$$

where $\beta_{x}=\beta_{y}=4, k_{x}=4$, and the center of wave packet is $x_{c}=-1$ and $y=0$. The initial wave packet has a momentum $\hbar k_{x}$ toward the product region, and the mass is $m=2000$ in this case. The incident wave function satisfying the initial condition and the free-space TDSE is given by $\Phi(x, y, t)=\Phi(x, t ; \beta=$ $\left.\beta_{x}, x_{0}=x_{c}, p_{0}=\hbar k_{x}\right) \Phi\left(y, t ; \beta=\beta_{y}, y_{0}=0, p_{0}=0\right)$, where the functional form of $\Phi(x, t)$ is given by Eq. (12), and this function was used in the source term for the integration of the modified TDSE.

In this case, the modified TDSE in Eq. (4) was solved using the fourth-order Taylor series method from $t=0$ to $t=1000$ with the time step $\Delta t=0.5$, and the required Taylor coefficients are evaluated by the automatic differentiation technique. This method has been employed to solve concentrationdependent diffusion problems, the Falkner-Skan equation, the nonlinear heat transfer problem, Schrödinger's equation, and the quantum hydrodynamic equations of motion [13-19]. The computational grid extends from $x=-1$ to $x=1$ with $\Delta x=0.025$ and from $y=-3$ to $y=3$ with $\Delta y=0.1$. Since the potential energy in Eq. (16) tends to a constant along each $x$ direction when $x$ goes to $\pm \infty$, we applied the outgoing wave boundary condition using the ratio method to the left and right boundaries. For example, for a grid point on the left boundary, the following equation was used to find the value of the scattered wave at point $(-1, y)$ from the previously computed values at two internal grid points, namely, $(-1+\Delta x, y)$ and $(-1+2 \Delta x, y)$ :

$$
\Upsilon(-1, y)=\frac{\Upsilon(-1+\Delta x, y)^{2}}{\Upsilon(-1+2 \Delta x, y)} .
$$



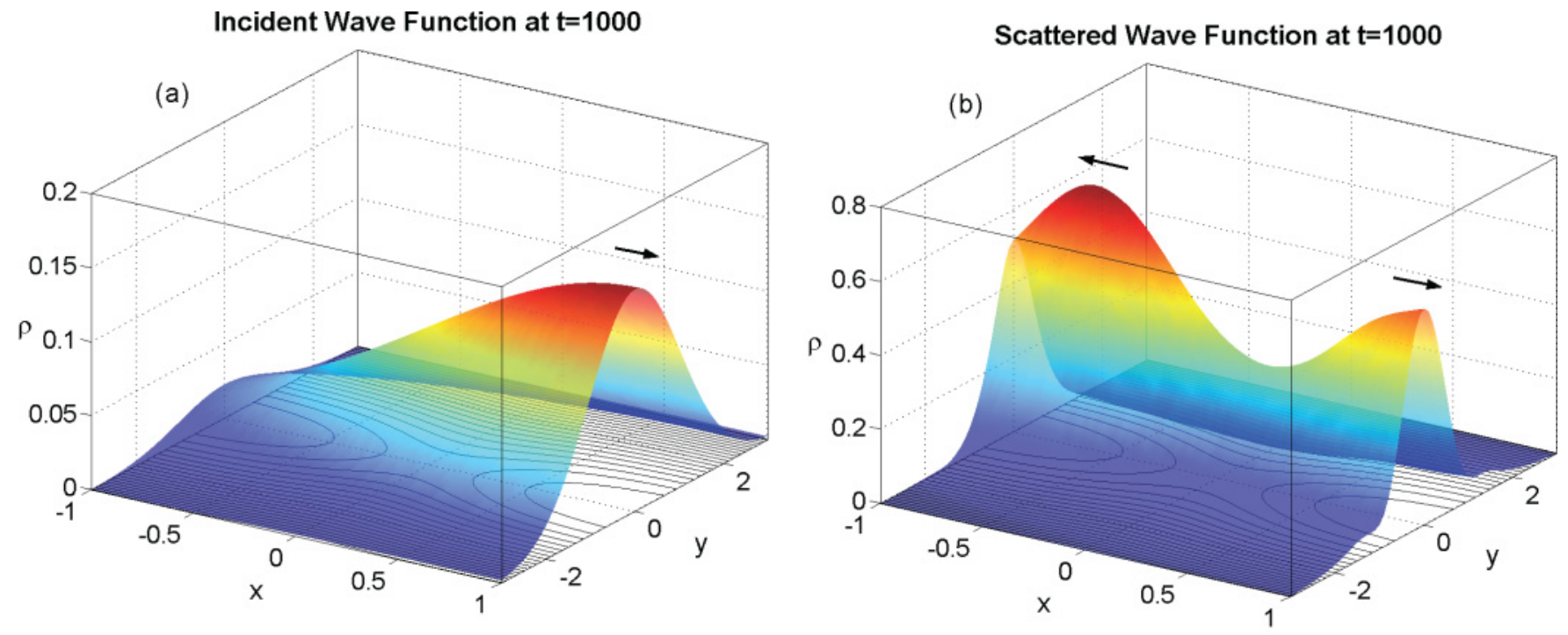

Total Wave Function at $\mathrm{t}=1000$

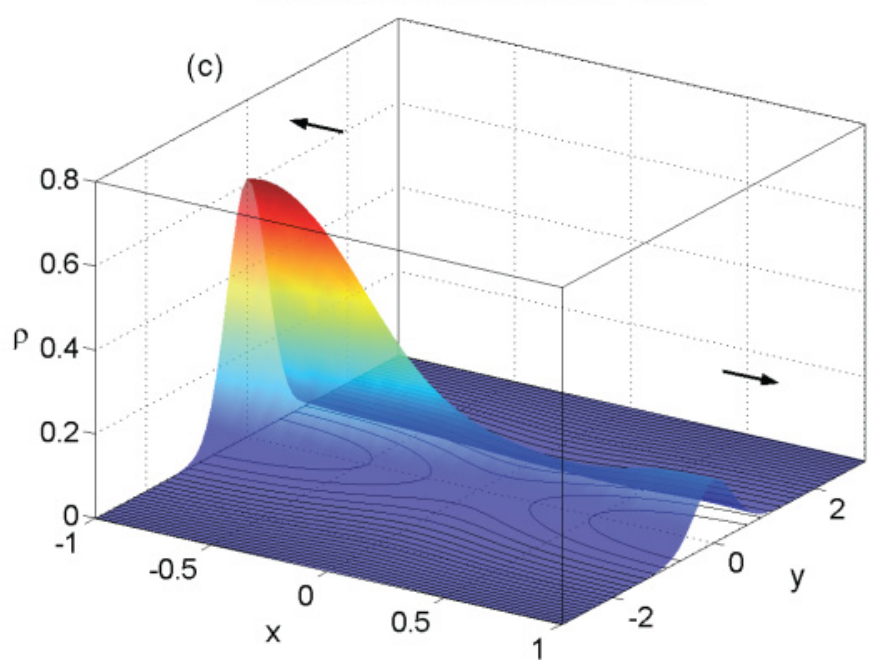

FIG. 4. (Color online) The incident, scattered, and total wave functions at $t=1000$ for $k_{x}=4$ with contours of the potential barrier. The arrows indicate the propagation directions of the wave packets.

On the other hand, since the potential energy in Eq. (16) gives a harmonic potential along each $y$ direction and it does not approach a constant at the top and bottom boundaries, we cannot apply outgoing wave boundary conditions to these boundaries. Thus, we require that the wave function vanishes at the top and bottom boundaries, and we need to use a wide range of the computational grid in the $y$ direction to avoid spurious reflections from these boundaries.

Figure 3 presents the incident, scattered, and total wave functions at $t=500$ for $k_{x}=4$ with contours of the potential barrier. Figure 3(a) shows that the incident wave is passing through the potential barrier. As shown in Fig. 3(b), the incident wave has interacted with the potential surface to generate the scattered wave. In Fig. 3(c), the total wave packet starts to split into the reflected and transmitted parts. In addition, Fig. 4 presents the incident, scattered, and total wave functions at $t=1000$. At this time, the incident wave has passed through the barrier. The scattered wave propagates through the boundaries without any reflection. Finally, the total wave packet has split into the reflected and transmitted parts, and a significant portion of these two wave packets has propagated through the boundaries. As shown in this figure, the transmitted wave packet consists of the incident and scattered waves, while the reflected wave packet originates mainly from the scattered wave.

Transmission probabilities can be obtained by integrating the probability density on the product side of the barrier $(x>0)$. Since we only obtain the probability density on a reduced computational domain, we cannot evaluate the transmission probability by integrating the probability density. However, analogous to the one-dimensional case, the time-dependent transmission probability can be obtained by the time integration of the probability flux along $x=0$,

$$
\begin{aligned}
P(t) & =\int_{0}^{\infty} \int_{-\infty}^{\infty} \rho(x, y, t) d x d y \\
& =\int_{0}^{t} \int_{-\infty}^{\infty} J_{x}\left(x=0, y, t^{\prime}\right) d y d t^{\prime}
\end{aligned}
$$




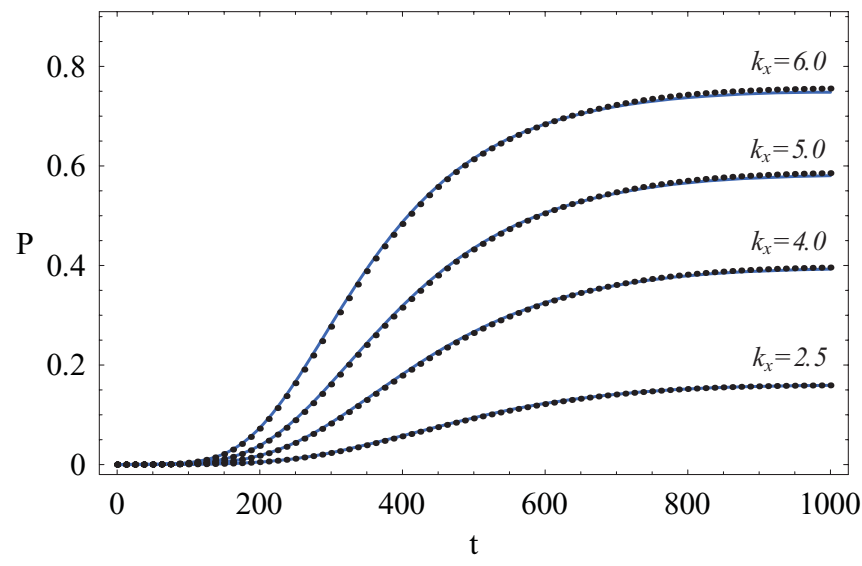

FIG. 5. (Color online) Time-dependent transmission probabilities obtained by the scattered wave formalism (dots) compared with the exact results (curves).

where the $x$ component of the flux is

$$
J_{x}(x, y, t)=\frac{\hbar}{m}\left[\Psi^{*}(x, y, t) \frac{\partial \Psi(x, y, t)}{\partial x}\right] .
$$

Figure 5 shows the time dependence of transmission probabilities for the initial wave packet with several values of $k_{x}$. Compared with the exact results obtained from the split-operator method using a large grid [20], these computational results are in excellent agreement with the exact results. Therefore, the scattered wave formalism not only significantly reduces the size of the computational domain, but also yields excellent time-dependent transmission probabilities with relative error less than $1 \%$.

\section{COMPUTATIONAL RESULTS FOR THE QUANTUM TRANSISTOR}

Using the scattered wave formalism, the time-dependent transport of a quantum wave packet in the conduction channel of the field effect transistor will be described. The three models differ in physical dimensionality, drain and gate voltages, and channel length. The electron potential energy in the channel is similar to that used in previous computational models of the field effect transistor, including Pinaud [21] (see Fig. 1), Fernandez [22] (see Fig. 2), Fu [23] (see Fig. 4), and especially Fu [24] (see Sec. IV and Fig. 1). For the first two models, the ratio method was used to implement outgoing wave boundary conditions and in the third model the polynomial fitting method was used. For all three models, comparisons were made between results obtained by imposing boundary conditions and accurate results obtained using large grids which did not employ the ratio or polynomial methods for imposing boundary conditions.

Because the amplitude and phase of total wave packet that enters the drain region are always smooth and free of oscillations, the boundary conditions were imposed directly on the total wave function. (In contrast, the amplitude of the scattered wave in this region may be oscillatory.) The scattered wave at the boundary point near the drain was then obtained by subtracting the initial wave function from the total wave function. However, the reflected wave near the source region usually has multiple oscillations. In this case, the boundary

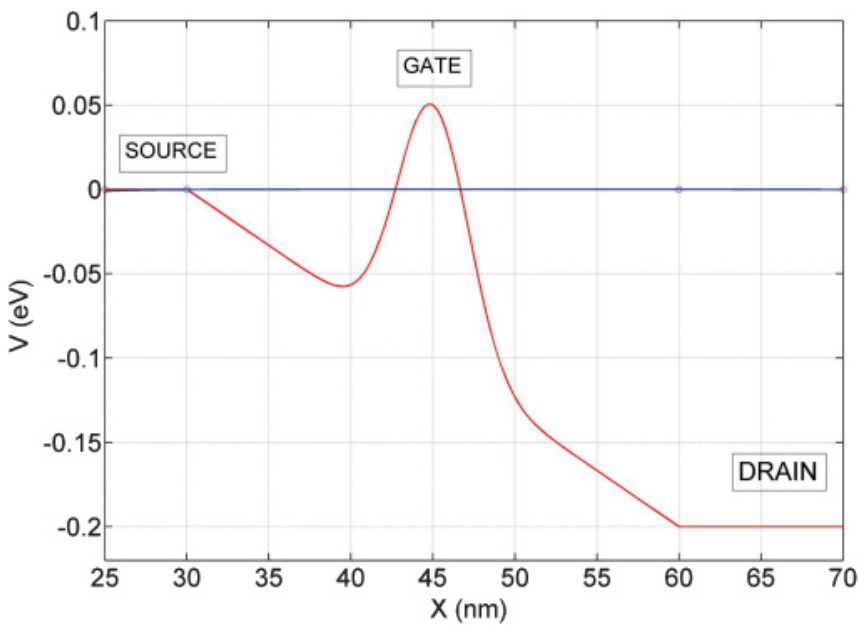

FIG. 6. (Color online) Potential energy for the one-dimensional transistor model. Outgoing wave boundary conditions were applied at $x=25 \mathrm{~nm}$ and at $x=70 \mathrm{~nm}$. The drain voltage is $0.2 \mathrm{~V}$, the gate voltage is $-0.15 \mathrm{~V}$, and the source region is assumed to be grounded, $V=0$. A Gaussian wave packet was launched into the conduction channel at $x=25 \mathrm{~nm}$.

conditions were imposed on the scattered wave. For these examples, the fourth-order Runge-Kutta method was used to integrate the modified TDSE for the scattered wave. The integration time step was $\Delta t=0.02 \mathrm{fs}$.

\section{A. Implementation of the ratio method for a one-dimensional model}

The potential energy for this one-dimensional (1D) model is shown in Fig. 6 . The $30 \mathrm{~nm}$ conduction channel extends from $x=30 \mathrm{~nm}$ to $x=60 \mathrm{~nm}$. Plateau regions with flat potentials were added on both sides of the conduction channel and outgoing wave boundary conditions (the ratio method) were applied at $x=25 \mathrm{~nm}$ and $x=70 \mathrm{~nm}$. The latter points are at the two edges of the computational domain, and in this region 181 grid points were employed (with the grid spacing $\Delta x=$ $0.25 \mathrm{~nm}$ ). The potential energy shown in this figure displays a downhill ramp leading from the source to the drain with a superimposed Gaussian barrier to represent the gate potential. A Gaussian wave packet was injected into the computational domain from the source region at the point $x=25 \mathrm{~nm}$.

Computational results are shown for the conduction channel at four times in Fig. 7. At each time, the total wave function computed from the incident and scattered waves (filled circles) is compared with the "exact" wave function (smooth curve) obtained by direct propagation of $\psi(x, t)$ on a large grid. For times up to about $1 \mathrm{ps}$, there is very good agreement. For this potential, oscillatory structure develops in the scattered and total wave functions in the vicinity of the dip in the potential energy (30 $\mathrm{nm} \leqslant x \leqslant 42 \mathrm{~nm})$.

\section{B. Implementation of the ratio method for a two-dimensional model}

The scattered wave method was applied to a twodimensional (2D) model for the quantum transistor. The potential energy in the conduction channel, shown in Fig. 8, extends from $x=30 \mathrm{~nm}$ to $x=57.5 \mathrm{~nm}$ and from $z=0 \mathrm{~nm}$ 

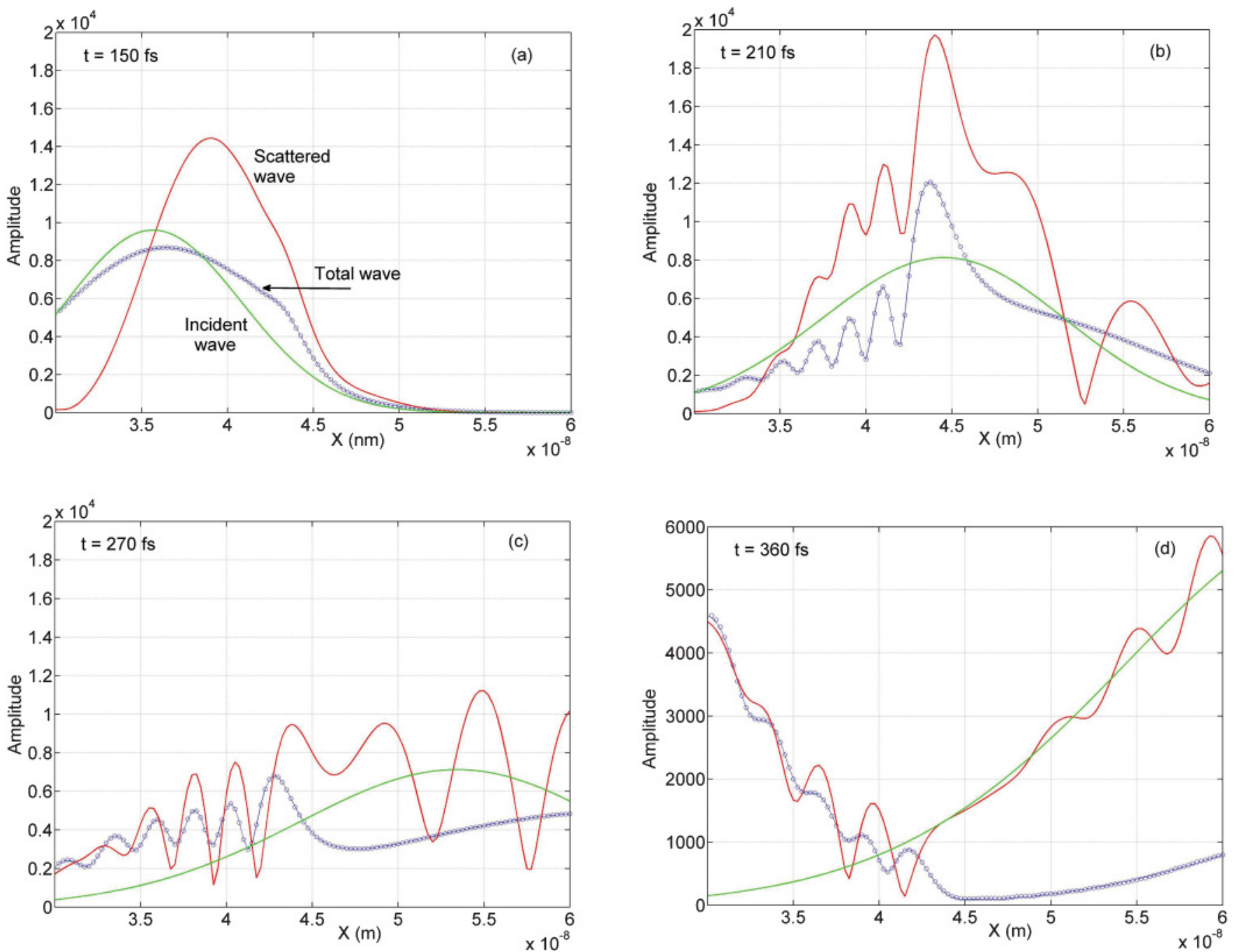

FIG. 7. (Color online) Wave functions for the 1D transistor model at four time steps. Amplitudes for the incident (green/light gray), scattered (red/dark gray), and total wave functions (dotted blue) are plotted. For the total wave function, results obtained using the ratio method to enforce outgoing wave boundary conditions (filled circles) are compared with accurate results (smooth curve) obtained on a large computational grid.

to $z=2 \mathrm{~nm}$. Plateau regions with flat potentials (not shown in this figure) were again added on both sides of the conduction channel, and outgoing wave boundary conditions (the ratio method) were applied at $x=20 \mathrm{~nm}$ and $x=70 \mathrm{~nm}$. The latter points denote the two edges of the computational domain along the $x$ coordinate. Within this 2D region, 1670 grid points were employed, with the grid spacing $\Delta x=\Delta z=0.25 \mathrm{~nm}$. Unlike Fig. 6, the potential energy shown in Fig. 8 displays an uphill ramp leading from the source to the drain and there is a superimposed Gaussian well to represent the gate potential. A Gaussian wave packet was injected into the computational domain along the line at $x=25 \mathrm{~nm}$.

Computational results for this model are shown at four time steps in Figs. 9 and 10. At each time, amplitudes of the scattered and total wave functions are displayed. In the first pair of plots, at $t=50 \mathrm{fs}$, the scattered wave is starting to form near the source region [see Fig. 9(a)] and the total wave function [see Fig. 10(a)] is dominated by the incident wave packet entering from the left side of the figure. In the second pair of plots, at $t=200 \mathrm{fs}$, the scattered wave has formed on both sides of the gate region [see Fig. 9(b)]

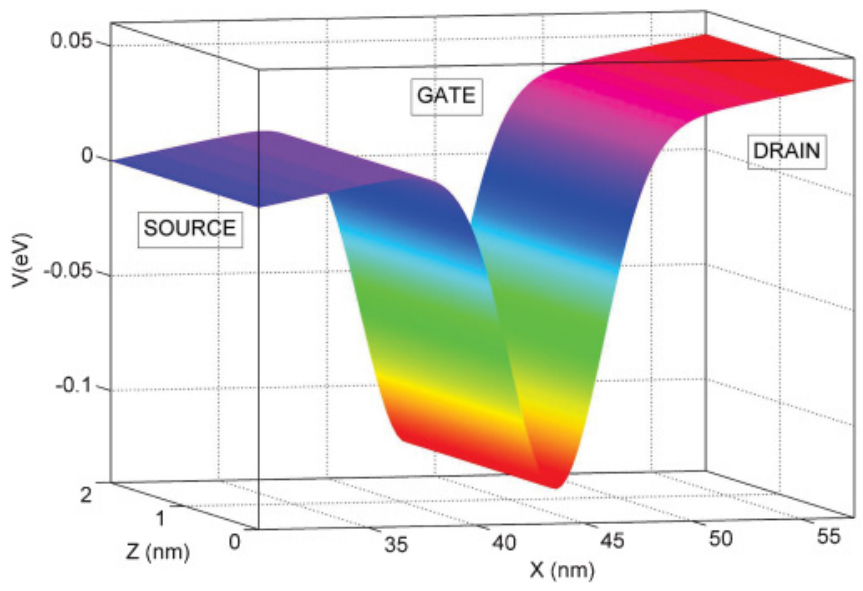

FIG. 8. (Color online) Potential energy for the two-dimensional transistor model. The drain voltage is $-0.05 \mathrm{~V}$, the gate voltage is $0.15 \mathrm{~V}$, and the source region is assumed to be grounded, $V=0$. A Gaussian wave packet was launched into the conduction channel from the source region on the left side of the figure. 

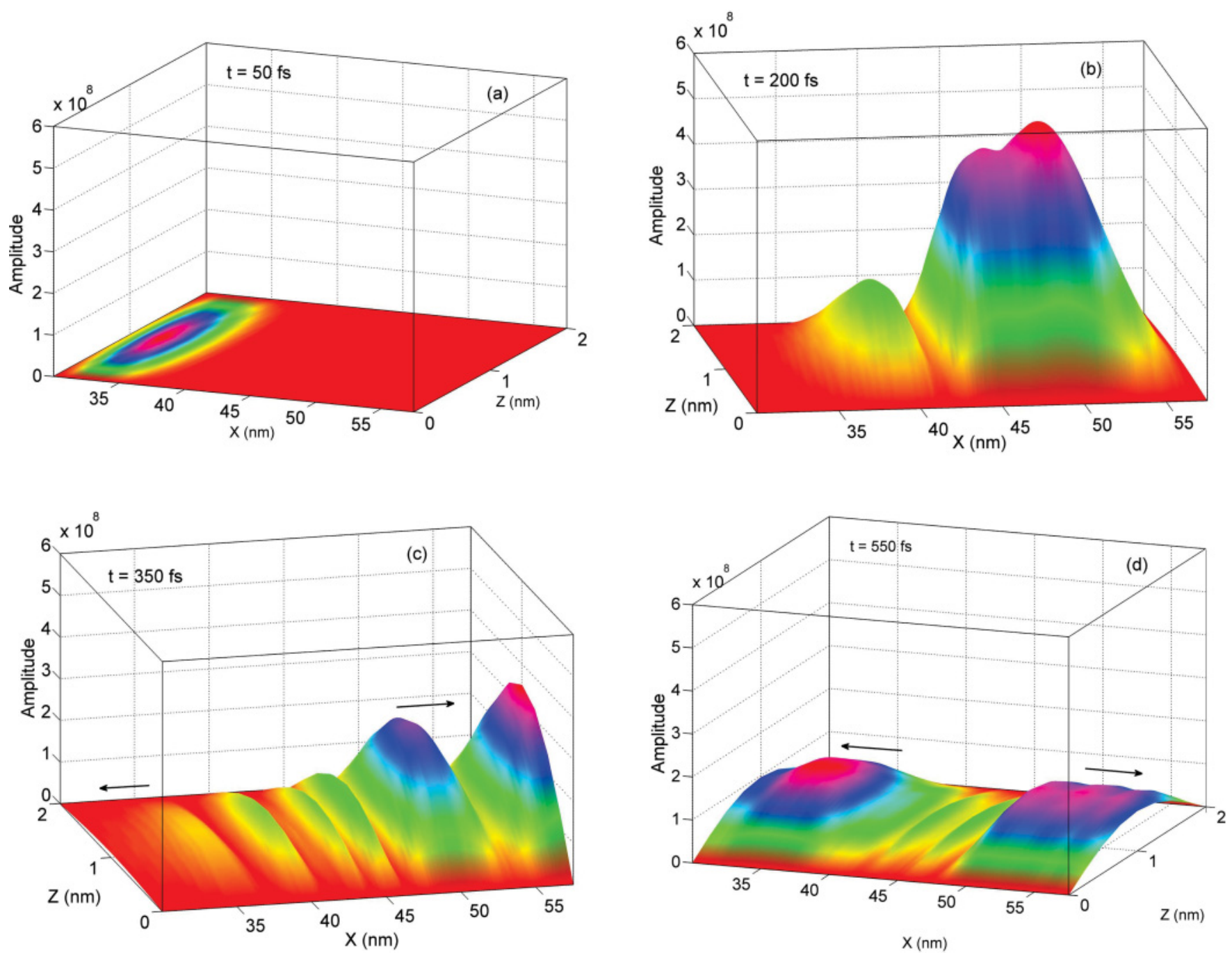

FIG. 9. (Color online) Amplitude of the scattered wave function, $|\Upsilon(x, z, t)|$, at four time steps for the two-dimensional transistor model. The arrows indicate the propagation directions of the wave packets.

and the maximum of the total wave function [see Fig. 10(b)] is approaching the drain on the right side of the figure. In the third pair of plots, at $t=350 \mathrm{fs}$, the scattered wave is exiting the channel region [see Fig. 9(c)] and the formation of a metastable state over the well in the gate region (between $x=$ $40 \mathrm{~nm}$ and $x=48 \mathrm{~nm}$ ) is evident in the total wave function [see Fig. 10(c)]. In the final pair of plots, at $t=550 \mathrm{fs}$, the scattered wave [see Fig. 9(d)] and the total wave [see Fig. 10(d)] continue to show the metastable state in the middle of each figure. In addition, it is noted that artificial ripples do not develop in either of these functions near the source and drain regions, where the boundary conditions are applied.

A measure of the accuracy of results obtained using the scattered wave formalism is shown in Fig. 11. This figure shows the time dependence of the integrated flux at three positions: near the source, at the gate, and near the drain. The integrated flux is defined by the integral over the transverse $z$ coordinate of the flux computed using the total wave function:

$$
F(x, t)=\int_{z=0}^{z=2} J_{x}(x, z, t) d z
$$

in which the $x$ component of the flux is computed from Eq. (20). The open circles in Fig. 11 are exact results obtained by propagating the wave function on a large grid and the dots denote results obtained with the scattered wave formalism. This figure shows excellent agreement between these two sets of results. This figure also shows that the peak of the flux enters the channel from the source at 90 fs and reaches the drain at $260 \mathrm{fs}$ after crossing the gate region at $170 \mathrm{fs}$. The flux entering the drain is free of oscillations, but the flux reflected back to the source shows two oscillations, at 600 and $750 \mathrm{fs}$. These oscillations occur about 150 fs after similar oscillations in the gate region.

\section{Implementation of the polynomial method for a 1D model}

The potential energy for this one-dimensional model is similar to that shown earlier in Fig. 6. However, the conduction channel extends from $x=30 \mathrm{~nm}$ to $x=80 \mathrm{~nm}$ and is longer than the one shown in this figure. Boundary conditions (the polynomial method) were directly applied at these two points. The gate and drain voltages are identical to those shown 

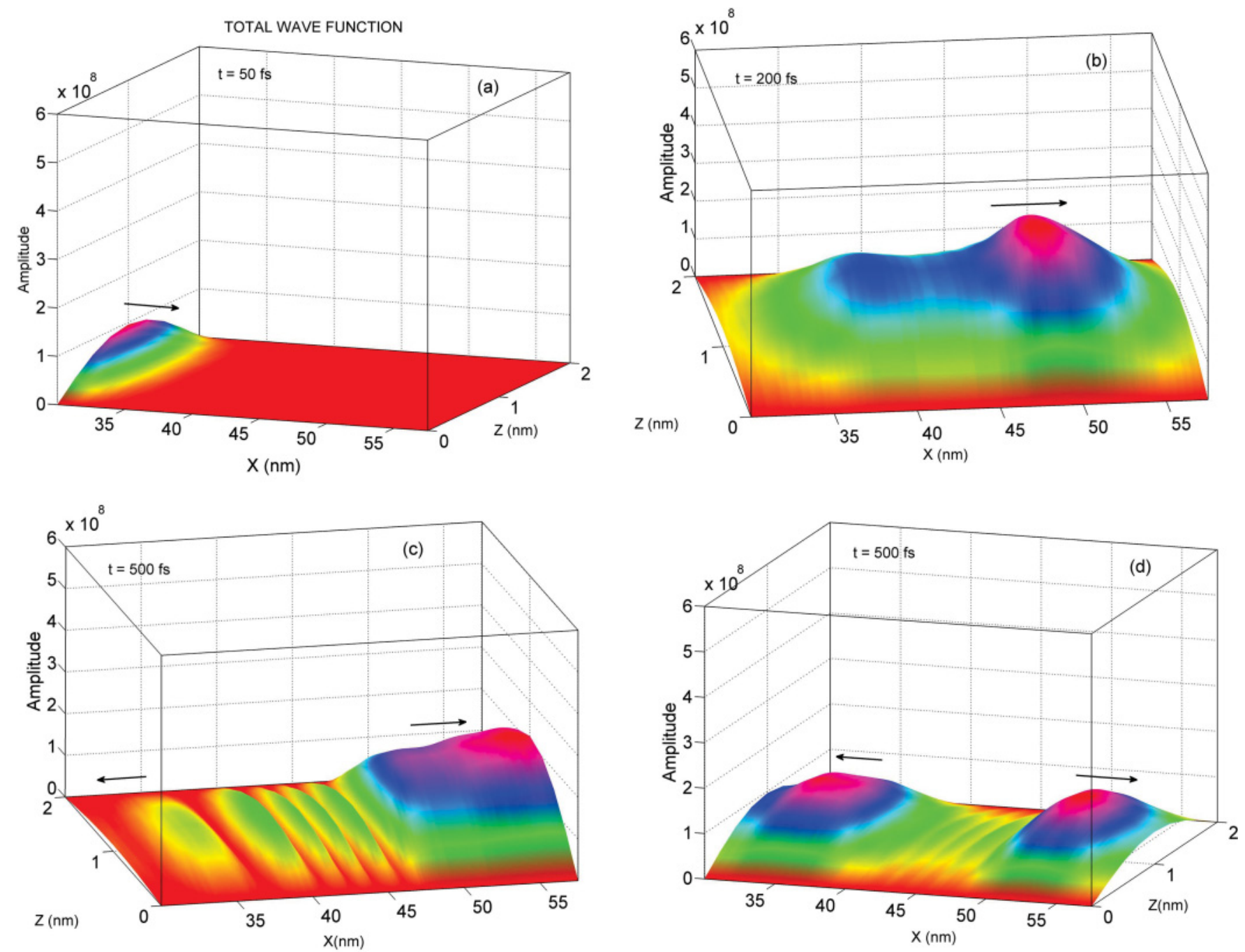

FIG. 10. (Color online) Amplitude of the total wave function, $|\Psi(x, z, t)|$, at four time steps for the two-dimensional transistor model. The arrows indicate the propagation directions of the wave packets.

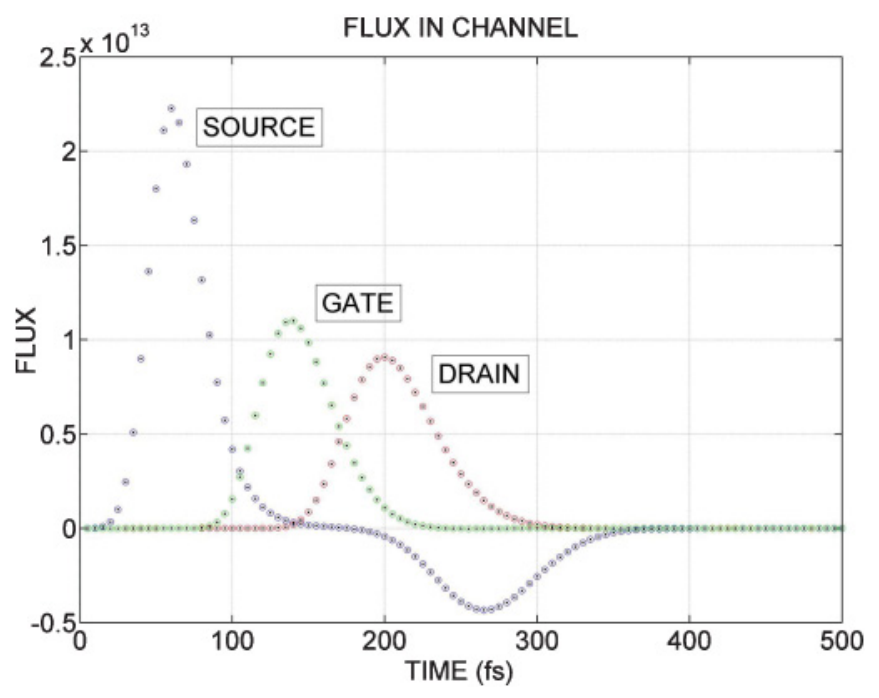

FIG. 11. (Color online) Time dependence of the integrated flux at three positions: near the source, at the gate, and near the drain. The open circles are exact results obtained on a large grid and the dots denote results obtained with the scattered wave formalism. in Fig. 6. A Gaussian wave packet was injected into the computational domain from the source region at the point $x=30 \mathrm{~nm}$ and the same integration method was used to propagate the scattered wave.

Computational results for this model are shown at three time steps in Fig. 12. At each time, the total wave function computed from the incident and scattered waves (filled circles) is compared with the exact wave function (smooth curve) obtained by direct propagation of $\psi(x, t)$ on a large grid. At these times, and for longer times up to $600 \mathrm{fs}$, there is very good agreement, with the largest relative errors being about $1 \%$. For times longer than about $300 \mathrm{fs}$, it was not possible to obtain results of this accuracy with the ratio method unless flat plateau regions were added on the left and right of the conduction channel to create an extended propagation zone.

\section{DISCUSSION AND CONCLUSIONS}

In this study, the scattered wave formalism was developed and applied to barrier scattering and quantum transistors. In this formalism, the total wave function is separated into the incident and scattered parts. The incident wave function can be 

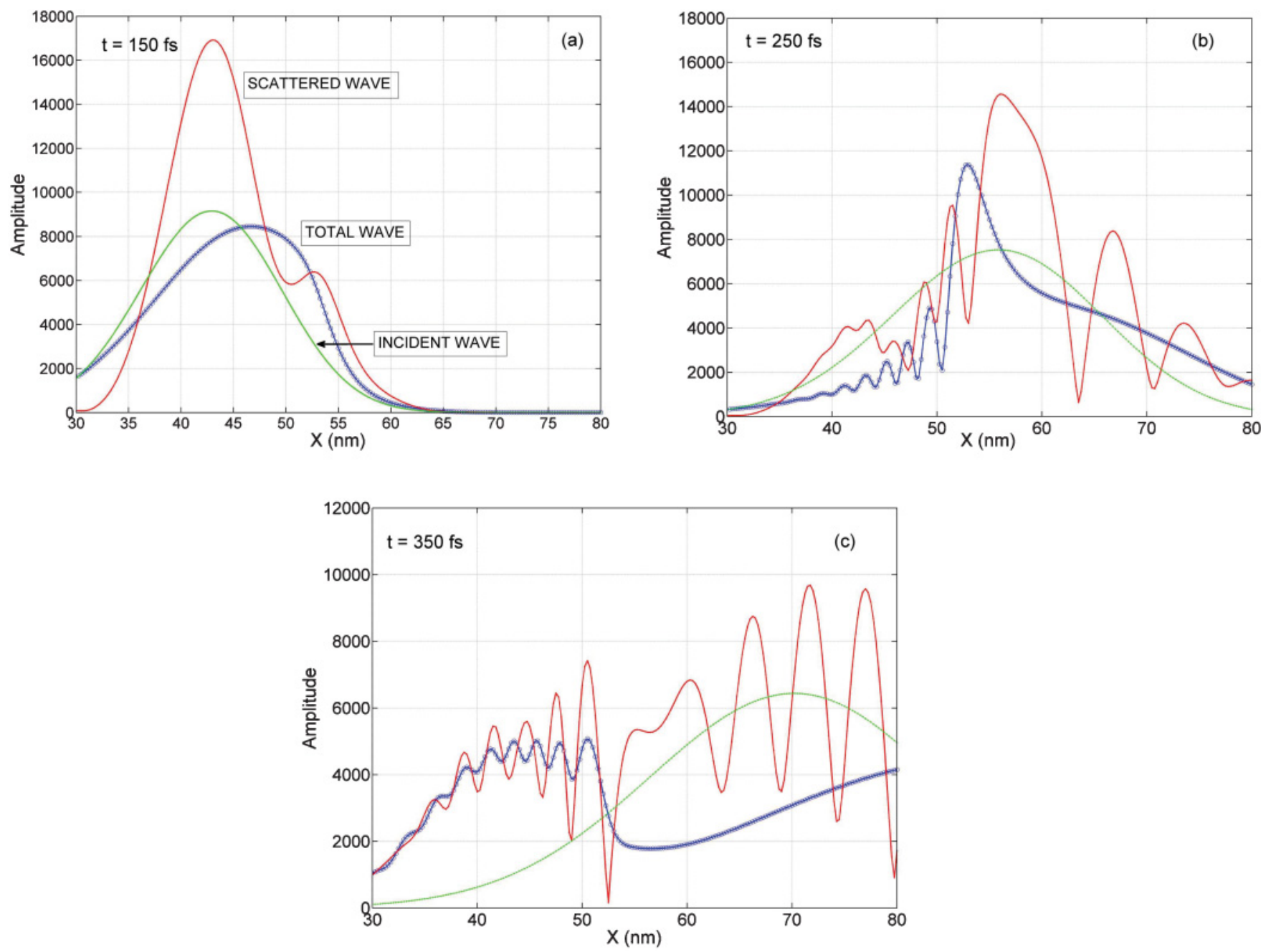

FIG. 12. (Color online) Wave functions for the 1D transistor model at three time steps. Amplitudes for the incident wave (green/light gray), scattered wave (red/dark gray), and total wave (dotted blue) are plotted. For the total wave function, results obtained using the polynomial method to enforce boundary conditions (filled circles) are compared with accurate results (smooth curve) obtained on a large computational grid.

determined either by analytically solving the free-space TDSE or by numerically solving the TDSE on a computational grid, and it serves as a source term in the modified TDSE for the scattered wave function. Then, the scattered wave function is solved on a reduced computational grid involving only the interaction region of the potential with outgoing wave boundary conditions. The outgoing wave boundary conditions can be handled either by the ratio method or by the polynomial method. At each time step, the scattered wave function at the interior grid points is employed to determine the scattered wave function at the boundaries.

The scattered wave formalism was applied to barrier scattering, including a general one-dimensional potential barrier with different asymptotic energies and a two-dimensional model system. We obtained the total wave function on a reduced computational grid and evaluated the time-dependent transmission probabilities by the time integration of the probability flux for one- and two-dimensional barrier scattering. For the two-dimensional system involving an Eckart barrier along the reaction coordinate coupled to a harmonic oscillator, outgoing wave boundary conditions were applied to the boundaries along the reaction coordinate with an asymptotically constant potential. For those boundaries with a nonconstant potential, because the rigid boundary condition was applied to the scattered wave function, the use of a large computational grid along this direction is generally required to avoid spurious reflections from the boundaries and to obtain accurate results.

On the other hand, the scattered wave formalism was also applied to the computational modeling of quantum transistors. The time-dependent transport of a quantum wave packet in the conduction channel of the field effect transistor was correctly determined by the scattered wave function with open channel boundary conditions at the interfaces between the device and the external contacts. In contrast with other open boundary methods, the scattered wave formalism can be readily applied to multidimensional systems (a three-dimensional quantum transistor model has been studied in Ref. [7]). In addition, compared with the exact results obtained by solving the TDSE on a large computational grid, the excellent computational results obtained in these examples demonstrate that the 
scattered wave formalism has wide applications to various time-dependent quantum processes and significantly reduces computational effort relative to other open boundary methods and the full-grid integration for the TDSE.

In this study, because the initial wave functions were chosen as Gaussian wave packets, the known analytical expressions of the time-evolving incident wave functions can be substituted into the source term in the modified TDSE for the scattered wave function. If the initial wave function is not a Gaussian wave packet or even the incident wave function is not known analytically, the incident wave can be propagated numerically in the left reservoir in a preliminary calculation. Then, the incident wave packet can be saved at the left boundary of the system. This information will be used as an injected function for the source term in the modified TDSE for the scattered wave function. In our previous study [7], this two-stage approach has been applied to the time-dependent transport of a quantum wave packet in the conduction channel of a three-dimensional field effect transistor.
The computational results presented in the current study illustrate that the scattered wave formalism allows accurate, efficient, and stable computations for long-time propagation for quantum dynamical precesses. The approximate outgoing wave boundary conditions developed in the current study require an asymptotically constant potential at the edges of the computational domain. Thus, the extension of the outgoing wave boundary conditions to a nonflat potential at the boundaries of the computational grid deserves further investigation. In the future, computational results for the channel current as a function of the drain and gate voltages for the nanotransistor will be analyzed, and the scattered wave formalism will be used to obtain self-consistent solutions of the TDSE and Poisson's equation.

\section{ACKNOWLEDGMENT}

We thank the Robert Welch Foundation (Grant No. F-0362) for their financial support of this research.
[1] C. Leforestier and R. E. Wyatt, J. Chem. Phys. 78, 2334 (1983).

[2] R. Kosloff and D. Kosloff, J. Comput. Phys. 63, 363 (1986).

[3] A. Vibók and G. G. Balint-Kurti, J. Chem. Phys. 96, 7615 (1992).

[4] J. R. Hellums and W. R. Frensley, Phys. Rev. B 49, 2904 (1994).

[5] M. Mangin-Brinet, J. Carbonell, and C. Gignoux, Phys. Rev. A 57, 3245 (1998).

[6] C. A. Moyer, Am. J. Phys. 72, 351 (2004).

[7] C.-C. Chou and R. E. Wyatt, Phys. Rev. Lett. 107, 030401 (2011).

[8] N. Carjan, M. Rizea, and D. Strottman, Comput. Phys. Commun. 173, 41 (2005).

[9] M. Rizea and N. Carjan, Rom. Rep. Phys. 60, 27 (2008).

[10] M. Rizea, J. Math. Chem. 48, 55 (2010).

[11] A. Goswami, Quantum Mechanics (Waveland Press, Long Grove, 2003).

[12] C. Pozrikidis, Numerical Computation in Science and Engineering (Oxford University Press, New York, 2008).

[13] A. Asaithambi, Appl. Math. Comput. 159, 641 (2004).
[14] A. Asaithambi, J. Comput. Appl. Math. 176, 203 (2005).

[15] A. Asaithambi, Appl. Math. Comput. 180, 264 (2006).

[16] A. Asaithambi, Appl. Math. Comput. 189, 943 (2007).

[17] A. Asaithambi, Appl. Math. Comput. 215, 4400 (2010).

[18] C.-C. Chou and R. E. Wyatt, Chem. Phys. Lett. 500, 342 (2010).

[19] C.-C. Chou and R. E. Wyatt, Int. J. Quantum Chem. 111, 4072 (2011).

[20] D. J. Tannor, Introduction to Quantum Mechanics: A TimeDependent Perspective (University Science Books, Sausalito, 2007).

[21] O. Pinaud, J. Appl. Phys. 92, 1987 (2002).

[22] E. Fernandez-Diaz, A. Alarcon, and X. Oriols, IEEE Trans. Nanotech. 4, 563 (2005).

[23] Y. Fu, M. Willander, and H. Pettersson, Appl. Phys. A 77, 799 (2003).

[24] Y. Fu and M. Willander, J. Appl. Phys. 97, 094311 (2005). 